\title{
La guerre et l'Etat libéral démocratique
}

\section{Vivienne Jabri}

Traducteur : Miriam Perier

\section{(2) OpenEdition Journals}

Édition électronique

URL : http://journals.openedition.org/conflits/2034

DOI : $10.4000 /$ conflits.2034

ISSN : $1777-5345$

Éditeur :

CCLS - Centre d'études sur les conflits lilberté et sécurité, L'Harmattan

Édition imprimée

Date de publication : 1 mars 2006

Pagination : 9-34

ISBN : 2-296-00622-1

ISSN : 1157-996X

Référence électronique

Vivienne Jabri, «La guerre et l'Etat libéral démocratique », Cultures \& Conflits [En ligne], 61 | printemps 2006, mis en ligne le 17 mai 2006, consulté le 30 mars 2021. URL : http://journals.openedition.org/ conflits/2034; DOI : https://doi.org/10.4000/conflits.2034

Ce document a été généré automatiquement le 30 mars 2021.

Creative Commons License 


\title{
La guerre et l'Etat libéral démocratique
}

\author{
Vivienne Jabri \\ Traduction : Miriam Perier
}

1 Les transformations de la modernité tardive sont souvent perçues comme les manifestations sociopolitiques et économiques des changements résultant des processus de mondialisation ${ }^{1}$. Mais de telles transformations doivent également être pensées en des termes permettant de comprendre leurs liens avec les formes émergentes de conflits internationaux. En relations internationales, le conflit, et la guerre en particulier, se situent dans le système anarchique que la pensée néo-réaliste envisage comme déterminant les relations entre Etats. La "troisième image » de Kenneth Waltz est ainsi systématique dans sa conception de la causalité de la guerre, rejetant tout mode de pensée qui placerait l'emphase sur les caractéristiques innées de l'être en tant qu'individu, ou le type de structure gouvernementale des Etats; respectivement la première et la seconde image ${ }^{2}$. Partant des prédilections de Waltz pour des explications systémiques de la guerre - des explications qui fournissent des hypothèses distinctes et se réfèrent aux rouages du système international ainsi qu'à la nature même de l'Etat comme unité de base de ce système -, on ne peut s'étonner qu'il ait été particulièrement critique envers les manifestations récentes de la guerre et du conflit que sont la "guerre au terrorisme" et l'invasion de l'Afghanistan ${ }^{3}$. Si on considère l'accent néo-réaliste mis sur la " continuité de la politique internationale », la réponse de Waltz ne nous surprend pas.

2 Face à ceux qui expriment une certaine nostalgie pour le monde des certitudes et des calculs de Waltz, ceux qui, comme nous, se situent du côté critique et constructiviste de la discipline peuvent interroger son approche épistémologique et ontologique. Les deux principaux défis qu'on pourrait soulever en réponse à Waltz se réfèrent, dans un premier temps, à la nature changeante du terrain que nous entendons comme « global », et, dans un second temps, à la signification des «types » d'Etats qui font les relations en politique internationale. Là où le monde waltzien est un monde d'uniformité et de prévisibilité, au sein duquel les Etats ne sont que des unités engagées 
dans des réponses calculées aux impératifs du système, l'arène globale est une arène d'incertitude et d'imprévisibilité, au sein de laquelle les Etats rivalisent pour se placer. Cette rivalité les oppose non pas entre eux, mais à des agences non étatiques, des corporations internationales aux organisations non gouvernementales, à des réseaux divers. Ce qui est le plus problématique pour Waltz et pour les Etats pensés comme uniformes, c'est que certaines de ces agences sont en concurrence avec l'Etat dans des domaines, qui, au sens weberien du terme, font partie du monopole étatique, notamment la sécurité et la violence ${ }^{4}$. Les transformations de cette ère moderne ne sont donc pas uniquement des transformations liées au niveau mondial - la troisième image de Waltz - mais elles ont une influence centrale sur la nature même de l'Etat moderne et sur la manière dont cet Etat moderne distinct réagit à ce qui constitue une menace aux attributions traditionnelles. La deuxième image de Waltz devient centrale dans cet âge des transformations globales, dans la mesure où l'Etat ne peut plus être pris comme une unité fermée, engagé de manière mécanique dans le calcul de ses intérêts distincts lorsqu'il est en concurrence avec des unités similaires dans les rouages tout aussi mécaniques d'un système d'Etats international.

Lorsque la deuxième image de Waltz prend de l'importance, les éléments constitutifs du mode de gouvernance, la machinerie bureaucratique de l'Etat, ainsi que la «sphère sociale » prennent sens dans nos considérations liées à la guerre et à la paix dans une ère mondialisée. Chacun à leur tour, ces éléments sont leurs manifestations transnationales; en d'autres termes, ils sont tous pris dans des réseaux de relations complexes qui rendent la division interne/externe très problématique. La spatialité des pratiques de gouvernement, ainsi que les relations qu'on associe traditionnellement à la société civile (que je nomme ici la «sphère sociale ») ne peuvent être considérées en termes d'insularité d'un ordre civique maintenu par le biais de technologies de contrôle et légitimé par des discours d'identité et d'affiliation. L'« intensification » des relations spatio-temporelles caractéristiques de l'ère de la mondialisation décrit non seulement une ère de connexion et de vulnérabilités mutuelles croissantes, mais modifie notre perception des dynamiques de guerre et de paix dans la modernité tardive. Ce faisant, de tels changements donnent une grande importance à la sphère sociale en elle-même, lorsqu'on ne considère pas uniquement les origines et les causes du conflit mais ses dynamiques et les réponses possibles. La spatialité - véritablement globale - de la sphère sociale rapproche les événements distants, de sorte que les différends traditionnellement associés aux conflits locaux, tels les conflits israélo-palestinien ou du Cachemire, acquièrent une portée mondiale et donc des réponses mondiales. Tandis que de telles réponses peuvent apparaître, par certains égards, institutionnelles, sous la forme des Nations unies ou de coalitions étatiques, elles peuvent également impliquer des acteurs de la sphère sociale globale avec leur propres expressions et articulations des conflits, offrant ainsi aux anciens conflits locaux des manifestations et des réarticulations mondiales. L'émergence de défis associés à la violence, elle-même liée à des organisations mondiales et souvent clandestines, exprime exactement ce à quoi nous faisons référence lorsque nous parlons de la spatialité de la sphère sociale.

Une autre manifestation de la spatialité mondiale de la sphère sociale émerge de la proximité des événements distants dans cette modernité tardive, et a, de nouveau, des implications sur nos considérations de la guerre et de la paix. Si la référence de Mary Kaldor aux «nouvelles guerres ${ }^{5}$ » peut être problématique par son cadre temporel, elle offre pourtant une caractérisation intéressante aux conflits comme ceux des Balkans et de l'Afrique sub-saharienne entre autres, en mettant en avant une fois 
encore les défis des nouvelles formes de guerre et les réponses qui y sont associées. Pour Kaldor notamment, le fait que les populations civiles soient très souvent les cibles de conflits de factions ou de la criminalité, et que ces initiatives soient soutenues par les réseaux de finances mondiaux et une loyauté ethnique représente de véritables défis aux droits de l'Homme, dans ce qui est considéré comme une arène mondiale de plus en plus cosmopolite. Pour Kaldor comme pour d'autres, dont Habermas ${ }^{6}$, le fait qu'un certain nombre de situations semblables, comme au Sierra Leone, en Bosnie, au Kosovo, en Somalie, pour n'en citer que quelques-unes, aient abouti à une intervention armée extérieure, suggère l'émergence d'une "nouvelle» forme de guerre, soit la "guerre humanitaire", là où les droits de l'Homme et leur protection deviennent essentiels. Le fait que les événements locaux ne puissent plus être isolés d'une vision globale se trouve au cœur des débats sur les responsabilités globales qui émergent de la spatialité globale de la sphère sociale.

5 L'implication de l'Etat démocratique libéral est centrale dans ces transformations qui affectent les manifestations modernes de la guerre et de la paix. Nous nous intéresserons donc, dans cet article, à l'impact que cette guerre de la modernité tardive - et spécifiquement la forme de guerre associée à la violence disperse des organisations en réseau - a sur l'Etat démocratique libéral. C'est dans ce sens que ladite deuxième image de Waltz nous intéresse tout particulièrement. En effet, alors que la théorie de la paix démocratique peut suggérer une zone de paix comprenant les démocraties libérales, les transformations mondiales auxquelles nous venons de faire référence mettent l'accent justement sur les éléments historiquement considérés comme constitutifs de l'Etat démocratique libéral. Là où l'ordre civique de l'Etat démocratique libéral est traditionnellement considéré comme une zone de paix, et comme la source de la paix entre les Etats, suivant la conception kantienne de la « paix perpétuelle ${ }^{7}$ », cet ordre civique se voit accorder une tout autre lecture par Michel Foucault et son analyse des rouages du pouvoir dans l'Etat moderne. L'objectif de cet article est donc d'explorer la lecture foucaldienne de la sphère sociale et de chercher à savoir comment cette lecture peut révéler les mécanismes du pouvoir dans la conjoncture historique actuelle, et tout particulièrement la manière dont les pratiques de guerre font partie de ces rouages. La dynamique à laquelle nous nous référons lorsque nous abordons l'idée de «matrice de guerre " révèle que, tandis que la politique internationale actuelle peut suggérer un certain bannissement des frontières et des distinctions, de telles démarcations sont néanmoins essentielles à la compréhension des défis posés à l'Etat démocratique libéral par les pratiques de la violence. Car, comme nous le verrons, bien que les frontières ne soient plus associées à des territoires distincts, elles sont " portées » dans la corporalité de l'Autre, et deviennent manifestes dans ces localisations transnationales que sont les rues, les voisinages, les villes, les pays de la modernité tardive, et dans ces localisations « entredeux ». La lecture foucaldienne des lignes de front qui place ces dernières au sein de la sphère sociale apparait particulièrement pertinente dans les manifestations modernes du conflit et de la violence, des pratiques qui défient tout particulièrement l'Etat libéral démocratique et sa propre perception comme lieu de la loi et des droits. L'article suggère que bien que l'analytique de la guerre et du pouvoir de Michel Foucault soit significative pour notre compréhension de l'actuelle « guerre au terrorisme » et de ses implications pour l'Etat libéral démocratique, elle doit être poussée à l'arène globale où la spatialité clairement globale de la sphère sociale est prise en compte. C'est en effet à ce niveau que la lecture foucaldienne de la souveraineté et de la gouvernementalité fait face à de véritables 
défis. C'est la mondialité des pratiques de guerre et de violence qui font de la guerre une technologie de contrôle.

La matrice de la guerre et le problème de la sécurité

6 La "guerre au terrorisme » est construite comme une guerre mondiale, transcendant l'espace et défiant manifestement les conventions internationales. Elle se distingue des guerres mondiales précédentes, y compris de la Première et la Seconde, en ce que ces deux dernières ont toujours été analysées par l'historiographie comme des confrontations interétatiques, bien qu'impliquant, de manière périphérique, des milices non gouvernementales, à certains moments et certains endroits. Des narrations futures de la confrontation actuelle et de ses différents paramètres reprendront bien sûr ces anciennes grilles de lecture. Mais ce qui fait tout l'intérêt de la présente discussion est l'aspect distinctement global de cette guerre, car il la rend particulièrement pertinente et topique pour des recherches qui s'attachent en premier lieu à la relation entre guerre et politique, entre la guerre et les processus politiques qui définissent l'Etat moderne. L'hypothèse initiale de cet article stipule que la guerre n'est pas confinée dans sa temporalité et sa spatialité propres mais qu'elle imprègne, au contraire, la normalité du processus politique, et qu'elle a donc une influence déterminante sur les éléments considérés comme constitutifs de politiques démocratiques libérales: responsabilité de l'exécutif, examen législatif, sphère publique de discours et d'interaction, égalité des citoyens devant la loi, et, pour rejoindre Habermas, légitimité politique fondée sur des pratiques de communication libres et égales (équitables) qui sous-tendent la solidarité sociale ${ }^{8}$. La guerre perturbe ces éléments et représente une période de crise et d'urgence. Une guerre permanente normalise clairement l'exceptionnel, inscrivant l'urgence dans les routines quotidiennes de la vie sociale et politique. Si les éléments de la guerre, le conflit, la fragmentation sociale, l'exclusion peuvent se poursuivre silencieusement au travers des assemblages de contrôle dans les sociétés libérales, l'itération persistante de la guerre dans la politique met ces pratiques au devant de la scène et porte en elle une refonte de la pensée sur la relation de la guerre à la politique.

7 La spatialité mondiale de cette guerre suggère des défis particuliers ayant un impact direct sur l'état libéral, ses obligations envers l'ensemble de ses citoyens et l'étendue de son implication sur l'atteinte à ses institutions. Ce serait toutefois une erreur de considérer que les pratiques mobilisées dans cette guerre mondiale sont de toute façon anathèmes à l'Etat libéral. Notre analyse argue que, bien qu'il soit crucial de reconnaître l'impact transformatif de la guerre au terrorisme, il est tout aussi important de rendre compte des continuités de la vie sociale et politique qui constituent les conditions de possibilité de cette guerre globale. Ces conditions ne sont pas simplement présentes ou apparentes d'un point de vue global, mais incorporent des pratiques locales profondément ancrées et institutionnalisées. La relation de renforcement mutuel entre les conditions locales et globales rend cette guerre distincte particulièrement envahissante, et potentiellement bien plus menaçante par ses implications pour les espaces disponibles à la contestation et la dissidence politiques.

La politique contemporaine mondiale est dominée par ce que nous pourrions nommer une "matrice de guerre " constituée d'une série de pratiques transnationales qui visent différemment les Etats, les communautés, et les individus. Ces pratiques impliquent les Etats en tant qu'agents, les bureaucraties étatiques et les organisations supranationales, des organisations quasi officielles et privées recrutées au service d'une 
machine globale hautement militarisée menée par les Etats-Unis, mais qui incorpore dans ses fins des alliances variées, toujours en flux. L'élément déterminant pour comprendre la matrice de la guerre est la notion de «pratique » qui comprend l'idée que toute pratique n'est pas simplement située dans un système de permissions et de contraintes mais qu'elle est elle-même constitutive de continuités structurelles, tant discursives qu'institutionnelles. Comme Paul Veyne l'écrit à propos de l'utilisation de ce terme par Foucault, «la pratique n'est pas une instance (comme le Ça freudien) ni un premier moteur (comme le rapport de production), et du reste, il n'y a chez Foucault ni instance ni premier moteur [...] ${ }^{10}$ » Il s'agit de ce sens récursif, selon lequel les pratiques (de violence, d'exclusion, d'intimidation, de contrôle, etc.) deviennent structurées dans les routines des institutions comme de l'expérience vécue ${ }^{11}$. Labelliser la guerre mondiale contemporaine en "guerre au terrorisme » confère à ces pratiques une certaine légitimité : elles sont dirigées vers l'élimination d'une menace directe. Alors que la menace de violences perpétrées par des réseaux clandestins à l'encontre des civils n'est que trop réelle et nécessite des réponses des Etats, ces dernières semblent présumer de larges attributions aux opérations, si larges que toute personne qui s'intéresse aux libertés associées à l'Etat démocratique ou aux droits des individus et des communautés, se doit de déchiffrer les implications de telles pratiques.

Les pratiques utilisées au nom de la sécurité, auxquelles nous venons de faire référence - souvent invoquées au nom de la liberté - sont, paradoxalement, situées dans une relation d'équilibre ${ }^{12}$. En effet, elles confèrent simultanément une primauté à la sécurité pour défendre les libertés, et peuvent faire l'objet de recherches en termes de discours de sécurisation (le pouvoir du discours agit sur la construction d'une menace, en même temps que celui des professionnels dans cette même construction ${ }^{13}$ ) ou en termes de discours de guerre, comme nous le verrons dans cet article. Les grammaires dont il s'agit sont liées entre elles, mais celle du discours de guerre est paradoxalement une grammaire critique, qui met en évidence les mécanismes du pouvoir et leurs imbrications avec la violence. Ce qui manque à la littérature de la sécurisation est une analytique de la guerre, analytique que nous souhaitons entamer ici.

Il nous semble que les pratiques précédemment citées constituent différents mécanismes de réponse face à ce qui est perçu comme une situation d'urgence au lendemain des attentats du 11 septembre 2001. L'invasion et l'occupation de l'Irak, l'incarcération sans procès de prisonniers dans des camps, en Afghanistan, à Guantanamo et dans d'autres lieux encore méconnus, l'utilisation de la torture contre les détenus, les assassinats extra-judiciaires, les détentions et les déportations, toujours sans procès, d'étrangers considérés comme une menace, des restrictions croissantes envers les réfugiés, leur enfermement dans des camps et des centres de détention, la construction en termes de sécurité du mouvement des personnes, et des restrictions des libertés publiques par des législations intérieures au Royaume-Uni, aux Etats-Unis et dans d'autres pays européens sont tous représentés dans les discours politiques comme des mesures de sécurité nécessaires à la protection de la société. Ce sont tout à la fois des mesures institutionnelles visant un Autre particulier, ennemi et source de danger.

11 On pourrait considérer que les pratiques mentionnées ci-dessus ne sont pas liées et qu'elles relèvent ainsi de modes d'analyses différents. Dans un premier temps, ces pratiques impliquent des agents différents et sont associées à des sujets différents. Les cas de l'Afghanistan et de l'Irak peuvent être mentionnés dans les discours comme des 
situations de guerre, et l'incarcération de réfugiés comme englobant des pratiques de sécurité. Mais ce qui lie ces éléments n'est pas tant le fait qu'ils constituent une taxonomie de pratiques différenciées. Ce qui les lie plutôt est l'élément d'antagonisme dirigé contre les Autres, distincts et particuliers. Une telle perspective suggère que les politiques de sécurité, qui comprennent la production de la peur ainsi que tout un ensemble de mesures d'exclusion, viennent au service de pratiques qui constituent la guerre et localisent le discours de guerre au coeur de la politique, non restreinte au national, mais de manière plus cruciale dans le contexte actuel, au niveau international. Les conséquences pour l'Etat de la modernité tardive et pour l'Etat libéral sont monumentales. Car une guerre perpétuelle à l'échelle mondiale a des implications pour les structures et l'action politiques, pour nos conceptions de la citoyenneté et du rôle de l'Etat dans sa réponse aux revendications de ses citoyens ${ }^{14}$, et pour les fonctionnements d'une sphère publique de plus en plus mondiale et donc de plus en plus multiculturelle. La matrice de la guerre est, comme nous le disions, principalement constituée autour de cet élément d'antagonisme. Cette notion d'antagonisme est associée à la menace existentielle. La présence continue de l'Autre constitue un danger, non seulement pour le bien-être de la société, mais également pour la poursuite de son existence dans la forme que ses membres connaissent. D'où la facilité relative avec laquelle des politiciens européens parlent de migrants d'origines spécifiques, comme représentants d'une menace à « l'idée de l'Europe » et à ses origines chrétiennes ${ }^{15}$. Il s'agit ici d'un discours d'exclusion culturelle et raciale fondé sur une certaine peur de l'autre. Alors que la guerre contre certaines organisations clandestines ${ }^{16}$ implique des opérations des deux côtés - ce qui peut se comprendre comme une guerre d'usure -, ce à quoi nous nous référons pour la matrice de la guerre est bien plus complexe, car nous avons là un ensemble de pratiques diffuses qui visent simultanément l'Autre typifié en termes culturels et raciaux et qui initient un domaine d'opérations plus large ayant un impact sur la société entière.

Les pratiques guerrières de l'immédiat après 11 septembre 2001 ont été combinées à des processus sociétaux reflétés dans les représentations des médias et dans la sphère publique élargie, où de plus en plus la source de menaces, source de terreur, est perçue comme l'Autre culturel, et plus particulièrement l'Autre, associé, d'une manière ou d'une autre, à l'islam, au Moyen-Orient et à l'Asie du Sud. Il y a alors une particularité à ce qu'Agamben nomme "l'état d'exception ${ }^{17}$ ", un état qui n'est pas tant généralisé et généralisable, mais un état expérimenté différemment par différents secteurs de la population mondiale. C'est précisément cette expérience différenciée de l'exception qui attire l'attention sur des pratiques aussi variées que la formulation de techniques d'interrogatoires par les services de renseignement militaire du Pentagone aux récentes dispositions (qui incluent des arrestations à domicile) des mesures antiterroristes au Royaume-Uni ${ }^{18}$, à des pratiques envahissantes très variées au sein de l'Union européenne, au discours de légitimation entourant l'invasion de l'Irak. Ce sont toutes des pratiques faisant appel à un discours de légitimation fondé sur la prévention et la préemption. Les ennemis construits dans les discours de la guerre sont donc toujours potentiels, toujours abstraits même lorsqu'ils sont identifiés, et partant, toujours élargis à une collectivité. Il y a ainsi un "profil » à l'état d'exception et son expérience. Les pratiques qui profilent certaines communautés, y compris les citoyens des Etats européens créaient des défis spécifiques à l'auto-compréhension de l'Etat libéral et de sa capacité à gérer la différence au XxI ${ }^{\mathrm{e}}$ siècle.

Bien qu'un certain nombre de mesures prises au nom de la sécurité - comme les propositions de mise en place de cartes d'identité au Royaume-Uni ou une surveillance 
accrue des transactions financières aux Etats-Unis - peuvent englober la population entière; la politique de l'exception a une signification raciale et culturelle ${ }^{19}$. Les personnes visées par des mesures exceptionnelles font partie de communautés raciales et culturelles spécifiques. La menace entendue, sous-jacente aux mesures précitées, est à présent ouvertement et différemment associée à l'islam comme idéologie, l'islam comme mode d'identification religieuse, l'islam comme mode de vie et de pratiques distinctes, et l'islam comme marque particulière associée à des organisations spécifiques qui épousent une certaine forme de retour à un califat islamique. Lorsque les pratiques sont informées d'un discours d'antagonisme, il n'y a pas de distinctions entre ces différentes formes d'identification individuelle et communautaire. Lorsqu'un profilage communautaire a lieu, la distinction entre, par exemple, le choix d'un mode de vie spécifique et celui d'une organisation spécifique disparaitt, et la diversité au sein de cette communauté profilée est sacrifiée au nom de certaines pratiques «de précaution » qui visent tous au nom de la sécurité ${ }^{20}$. Lorsque les pratiques et le langage de l'antagonisme sont inscrits dans le racialisme et le culturalisme, ils placent la culpabilité au cœur de la communauté identifiée tout entière, de sorte que les membres individuels de cette communauté ne puissent plus simplement être citoyens d'un Etat séculier et multiculturel, mais sont constitués dans les discours comme des citoyens particuliers, sujets à des pratiques particulière et, donc, exceptionnelles. Lorsque le ministre de l'Intérieur du Home office déclare que les membres de la communauté musulmane doivent s'attendre à être arrêtés par la police, il exprime simplement la situation présente, selon laquelle la communauté musulmane est particulièrement vulnérable à une vigilance et à des mesures envahissantes qui ne s'appliquent pas au reste des citoyens ${ }^{21}$. Nous savons également qu'un profilage clairement racial a lieu, de sorte que ceux qui sont physiquement profilés soient sujets à des mesures exceptionnelles.

14 Le régime démocratique a toujours défini les limites de ses politiques en termes de frontières de l'Etat. Il existe un contrat social selon lequel le rôle de l'Etat est de protéger contre tout danger venant de l'extérieur. Ce qui appartient à cet ordre contractuel est la «société civile» avec ses relations de droits et d'obligations, tandis que toute relation à l'extérieur dépend des résultats changeants de la diplomatie. Dans cette conception libérale du social et du global, dont les fondations viennent de Kant ou de Hobbes, la guerre est une condition permanente qui menace l'Etat et son ordre interne pacifique, bien que cet ordre soit imposé par des moyens voulus rationnellement. Ce qui importe particulièrement, en relation à l'Etat moderne que Hobbes a ordonné, est que les frontières se sont de plus en plus réduites et que nous sommes témoins de l'effilochage des politiques démocratiques, un effilochage qui expose les tensions inhérentes aux pratiques des gouvernements libéraux.

La matrice de la guerre est configurée en termes d'antagonisme, et l'antagonisme implique la présence de démarcations, ou, pour être plus précise, de l'inscription de limites pour différencier les amis des ennemis. Cette frontière est différente de celle de l'Etat, de sorte que même si les frontières d'un Etat sont d'une grande importance en termes migratoires, il serait toutefois naïf de considérer que la frontière est une affaire simplement liée aux douanes et aux règles liées aux mouvements des peuples. Dans le contexte actuel de relations socio-économiques mondialisées, le mouvement des biens et des personnes est en effet largement facilité aux frontières de l'Etat. La frontière prohibitive, celle qui inscrit les amis et les ennemis, n'est pas contiguë à l'Etat mais se retranche toujours plus vers l'intérieur. Ce faisant, elle relocalise toute distinction 
pouvant être faite entre un ordre « civil » interne, et la guerre potentielle à l'extérieur. Dans ces conditions, la guerre vient à pénétrer la société, amenant avec elle les éléments qui constituent la figure de la guerre, notamment l'inimité et l'antagonisme, la peur et la haine de l'autre.

Même lorsque ladite guerre au terrorisme ne reconnaît aucune frontière comme limite à ses pratiques - en effet, nombreuses de ses pratiques se font dans des espaces transnationaux et parfois indéfinissables -, il est essentiel de comprendre que cela ne signifie pas que les frontières ne sont plus construites ou qu'elles ne débordent pas sur la sphère du politique. Le paradoxe du contexte actuel est que, tandis que la guerre au terrorisme dans toutes ses manifestations assume une arène illimitée, les frontières et les démarcations sont au cœur de ses opérations. Il est important de souligner que ces démarcations ainsi que les pratiques d'exclusion qui les soutiennent ne sont pas contiguës à celles de l'Etat. On pourrait plutôt dire d'elles qu'elles sont localisées et perpétuellement construites sur la corporalité de ceux qui sont construits comme ennemis, comme menace à la sécurité. C'est en effet le retrait corporel de tels sujets qui est au cœur de ce qui est construit comme des mesures antiterroristes, typifiées dans les pratiques de guerre directe, dans l'utilisation de la torture, dans des incarcérations extra-judiciaires, et dans des lieux de détention autorisés par la loi. On pourrait ainsi se demander si de telles mesures constituent la violence ou les relations de pouvoir, lorsqu'en suivant Foucault nous considérons que le premier agit sur les corps dans le but de blesser, alors que le second agit sur les actions des sujets et assume, comme le suggère Deleuze, une relation de force et donc un sujet qui peut agir ${ }^{22}$. Nous souhaitons montrer ici que la violence est imbriquée dans les relations de pouvoir, qu'elle est un mode de contrôle, une technologie de gouvernementalité. Lorsque la population irakienne est visée par des bombardements aériens, la conséquence dépasse la blessure et cherche la pacification du Moyen-Orient comme région politique.

17 Lorsque des mesures législatives et bureaucratiques sont mises en place au nom de la sécurité, ce sont des catégories de population qui sont visées. En même temps, la guerre au terrorisme et les discours sécuritaires utilisés dans sa légitimation sont conduits et construits en des termes qui impliquent la défense ou la protection de populations. Une option consiste à limiter les efforts policiers, militaires et de renseignement en visant des organisations spécifiques. Mais, ce sont la construction illimitée de la guerre au terrorisme et son orientation envers des communautés raciales et culturelles particulières qui sont au cœur du défi posé à l'Etat libéral démocratique. Dans des conditions construites en termes d'urgence, la guerre s'introduit dans les discours politiques, de sorte que ces derniers soient sujets aux restrictions et aux impératifs de la guerre et des pratiques constitués en termes de demande de sécurité contre une menace existentielle. Les conséquences pour les politiques démocratiques libérales et pour nos conceptions de l'Etat moderne et ses institutions ont une grande portée ${ }^{23}$, car le régime démocratique libéral qui se considère en guerre perpétuelle est également en état de mobilisation permanente - état dans lequel tous les aspects de la vie publique sont adaptés en fonction du combat contre des ennemis potentiels, internes ou externes.

18 La plus grande leçon que nous tirons des écrits de Michel Foucault est que la guerre, ou, comme il l'écrit «le grondement de la bataill $e^{24}$ » n'est jamais bien loin de la gouvernementalité libérale. Si nous les concevons dans les termes foucaldiens, la guerre et les mesures antiterroristes sont perçues non pas comme discontinues par 
rapport aux gouvernements libéraux, mais comme issues des conditions que le gouvernement libéral et l'Etat moderne ont historiquement mis en place. En lisant les analyses de Foucault sur l'émergence de la société disciplinaire, nous voyons la continuité de la guerre dans la société et, non pas comme chez Hobbes ou d'autres dans l'histoire de la pensée, l'idée selon laquelle la guerre a lieu à l'extérieur de la société et de son ordre civil. La société disciplinaire n'est pas simplement une accumulation de procédures institutionnelles et bureaucratiques qui pénètrent le quotidien et la routine; elle a, traversant ses interstices, les éléments constitutifs de la guerre comme continuité, ce qui comprend la confrontation, la lutte et le retrait physique de ceux que l'on considère comme les ennemis de la société. Dans Il faut défendre la sociétéé et le premier volume de Histoire de la sexualitée ${ }^{26}$, référence est faite aux continuités qui structurent la guerre dans la société, une structuration qui est discursive et institutionnelle ${ }^{27}$. La référence au " grondement de la bataille » suggère la confrontation et la bataille, la construction omniprésente de la menace qui revient à l'autre particulier-spécifique, l'immédiateté de la menace et la construction de la peur de l'ennemi, et, ultimement, le retrait physique de l'ennemi comme source de peur. L'analytique de la guerre embrasse également les techniques des militaires et leur présence dans la sphère sociale, en particulier le contrôle et la régulation des corps, une précision minutée et une instrumentalité qui transforme une machine de guerre en machine active et tueuse. Il y a donc dans la matrice de la guerre le niveau du discours et le niveau des pratiques institutionnelles. Les deux sont liés par un rapport mutuel d'implication et de permission. Il y a également le niveau des corps et celui de la population. Pour Foucault :

«le biologique se réfléchit dans le politique; le fait de vivre n'est plus ce soubassement inaccessible qui n'émerge que de temps en temps, dans le hasard de la mort et de sa fatalité ; il passe pour une part dans le champ de contrôle du savoir et d'intervention du pouvoir. Celui-ci n'aura plus affaire seulement à des sujets de droit sur lesquels la prise ultime est la mort, mais à des êtres vivants, et la prise qu'il pourra exercer sur eux devra se placer au niveau de la vie elle-même $[. .].]^{28}$ ».

19 Ce qui précède suggère l'idée de la guerre comme continuité dans la vie sociale et politique. La matrice de la guerre suggère à la fois des pratiques discursives et institutionnelles, des technologies qui visent corps et populations, édictées dans un réseau complexe de localisations. Le moment critique de cette forme d'analyse est de montrer que la guerre n'est pas simplement une occurrence isolée qui aurait lieu comme forme d'interruption d'un ordre pacifique existant, mais, bien plus, que cet ordre pacifique est imbriqué dans les éléments de guerre, présents comme continuités dans la vie sociale et politique, des éléments qui sont profondément ancrés et qui permettent l'actualité de la guerre dans son sens traditionnel de champ de bataille. Ceci implique une continuité des genres entre le disciplinaire, le carcéral et les manifestations violentes du gouvernement.

Selon cette vision foucaldienne, un tel gouvernement est imprégné des pratiques de guerre discursives et institutionnelles comprenant l'antagonisme, la construction d'ennemis, la perception d'une menace existentielle, des alliances et des inimitiés, le désir de retirer ou d'oblitérer la source de la menace. Dans ce mode analytique, la guerre n'est plus perçue comme interruption d'un ordre pacifique. Elle est plutôt omniprésente dans les pratiques discursives et institutionnelles qui constituent le monde social. L'analytique de la guerre de Foucault permet une réflexion critique sur le présent, car elle révèle des pratiques gouvernementales au travers du prisme de la 
guerre, révélations qui pointent immédiatement au contrôle des corps et des populations, et avant tout les moyens par lesquels les discours de la guerre génèrent des subjectivités particulières.

Guerre globale et transformation de l'Etat libéral

21 Si l'on utilise la lecture foucaldienne de la guerre pour analyser la sphère sociale, cela revient à suggérer la présence perpétuelle de la guerre dans la société. Il n'y a, en un sens, pas de lieu qui soit exempt de relations de pouvoir, et, par conséquent, de guerre et de relations de force. Alors que Foucault déplace son analytique du pouvoir hors de la guerre comme outil d'analyse de la sphère sociale, son interprétation du pouvoir reste influencée par ce qu'il considère comme étant les éléments de la guerre. On pourrait en effet reprocher à Foucault de ne pas avoir su distinguer les différents états, ou plutôt, les différentes formes de résistance. Comme nous l'évoquions en introduction, tout comme pour Kenneth Waltz le pouvoir est la continuité de la politique internationale, pour Michel Foucault le pouvoir est la continuité de la sphère sociale. Ceci implique que toute réalisation historique en termes de transformations de la condition humaine, par exemple, dans la capacité de la société civile à résister aux excès du pouvoir étatique, est compris, en termes fonctionnels chez Foucault; la production de sujets « libres » sert les mécanismes de la gouvernementalité libérale ${ }^{29}$.

22 La distinction finalement établie par Foucault entre violence et pouvoir est cruciale. Ainsi, tandis que la violence cherche l'oblitération du sujet, le pouvoir assume la présence continue du sujet, un sujet qui peut contester le pouvoir et la domination ${ }^{30}$. Néanmoins, même lorsque Foucault établit cette distinction, sa conception de la gouvernementalité libérale est informée, comme nous l'avons précédemment stipulé, par une analytique de la guerre, le monde social est ainsi toujours imbriqué à la figure de la guerre, «le grondement de la bataille ». De ce point de vue, les actions entreprises par les Etats libéraux démocratiques en réponse au terrorisme global sont simplement une continuité de pratiques qui définissent et soutiennent la gouvernementalité libérale.

Il est toutefois important de souligner que c'est justement cette gouvernementalité qui a historiquement généré des mécanismes par lesquels la capacité à être des sujets souverains est inscrite dans le droit, quelque peu protégé des instincts autoritaires de l'Etat. La souveraineté se voit diminuée et cède historiquement la place à la gouverne(mentalité) des populations et donc au développement de régimes respectant les droits de l'Homme, des pratiques d'Etat-providence, des conventions contre la torture et le génocide, et d'autres formes de mesures globales qui ont pour but la protection des individus et des populations contre les excès du pouvoir de l'Etat ou du marché. La guerre a toutefois été une condition perpétuelle, non pas dans son sens clauzewitzien, mais dans une manifestation plus diffuse, transnationale. Tout comme la mondialisation des relations socio-économiques a des implications sur l'émergence d'une violence transnationale, cette émergence transforme à son tour l'arène mondiale. Ce lien entre la mondialisation et la violence de réseaux internationaux est essentiel dans la compréhension des implications profondes que l'émergence de la violence transnationale a eues sur le régime libéral et ses institutions. Pour rendre l'image foucaldienne internationale, il faut recentrer l'attention sur la violence comme forme de contrôle. Il s'agit, de plus, de recentrer l'attention sur la localisation de l'espace politique et les implications de ses ré-articulations transnationales. Là où finalement Foucault détourne son 
attention de la violence, les transformations mondiales actuelles recentrent l'attention sur la violence et les défis actuels subis par l'Etat moderne libéral et ses institutions.

L'ouvrage récent de Hardt et Negri, Multitude: War and Democracy in the Age of Empire, met l'accent sur ces transformations, et en particulier, sur le passage d'une forme de guerre à des formes d'actions dispersées mais à portée mondiale. Ce qui nous intéresse essentiellement est l'attention particulière portée par ces auteurs sur l'aspect transnational de la guerre de la modernité tardive et de l'émergence de structures juridico-politiques supranationales impliquées dans la légitimation de pratiques violentes via des invocations de l'Humanitée ${ }^{31}$. L'analyse de Hardt et Negri insiste sur un domaine spatial mondial et systémique. Les guerres sont ainsi transformées en opérations policières et suggèrent que " la présence constante d'un ennemi et de la menace sont nécessaires pour légitimer une violence impériale ${ }^{32} »$.

Mais Hardt et Negri se trompent en observant le présent uniquement en termes de "violence impériale ». En effet, bien que des éléments de la situation actuelle puissent être décrits comme une violence impériale (l'invasion et l'occupation de l'Irak par exemple), les pratiques variées auxquelles nous avons fait référence, comme la "matrice de la guerre ", sont bien plus complexes, car elles pénètrent les sociétés au travers des espaces géographiques, l'Occident libéral y compris. La littérature sociologique fait le lien entre les transformations mondiales - en particulier les mondialisations de la vie sociale et politique - et l'émergence du danger et du risque. Lorsque le domaine spatial est conçu comme ayant une portée mondiale, il suggère des espaces indéterminés qui défient quelque peu l'ordre et le contrôle, qui transcendent l'espace et le temps et, ce qui importe dans le contexte actuel, qui soient une source de risque et de danger. Les transformations de la modernité tardive sont donc structurelles et phénoménologiques. De telles transformations sont considérées comme mondialement visibles, incorporant dans leur sphère des expériences vécues partout. Ceci suggère des transformations institutionnelles et de l'expérience, visibles dans la conduite de la guerre et dans les discours de sa légitimation. Mais avant tout, il y a la perception que les transformations mondiales associées à la modernité tardive ont des ramifications dans l'Etat, la souveraineté, et la localisation de l'autorité politique ${ }^{33}$. Tous ces écrits insistent sur le fait que les transformations de l'espace politique présentent une source d'insécurité à tous les niveaux d'interaction sociale. L'hypothèse implicite est celle selon laquelle les mouvements au-delà des confins de l'Etat ont engendré une modification historique et généralisée dans l'auto-compréhension des sociétés, une modification qui a engendré à son tour un sens d'insécurité et de danger existentiel.

De telles analyses ne parviennent de nouveau pas à nous renseigner sur la spécificité de la relation entre la violence et les opérations de pouvoir actuelles. Tout comme les transformations qu'elles décrivent peuvent contenir des sources d'insécurité et de risque, ces mêmes dynamiques peuvent être construites comme des sources potentielles de liberté et de richesse accrues ${ }^{34}$. Toutefois, il est important de noter que de telles hypothèses ont vu leur force grandir au lendemain du 11 septembre 2001. Zygmunt Bauman, critique sur les réponses à ces événements, suggère que le 11 septembre constitue "une fin symbolique de l'ère de l'espace », mettant l'accent sur l'élément "symbolique» en ce que "les explosions des tours du World Trade Centre à Manhattan ont mis au coeur de l'attention publique certaines transformations souterraines et graduelles qui étaient en cours depuis un bon moment déja ${ }^{35}$ ». Pour Bauman, le mondial est 
un terrain irrégulé, "non-colonisé, politiquement incontrôlé, profondément dérégulé, extraterritorial », et, par là, il est la source d'insécurité qui construit dans son sillage un «espace mondial» qu'est le "nouveau pays-frontière" ("new frontierland») où les adversaires sont « extraterritoriaux ». Ceux qui vivent dans cet espace indéterminé, en l'occurrence les réfugiés, deviennent les cibles sur lesquelles "l'insécurité existentielle ", associée au "pays-frontière " mondial et construite en tant que telle par les hommes politiques, peut être "condensée, déversée et dispersée $e^{36}$. Au sein de ce "pays-frontière " mondial ou planétaire, l'étranger devient la cible première, non seulement dans les pratiques qui visent les réfugiés, mais aussi dans celles qui incarcèrent et déportent l'étranger sans respect du droit au nom de la sécurité (mondiale).

Bien que la notion de "pays-frontière " mondial de Bauman soit suggestive, elle ne parvient pas à aider à la compréhension des conditions qui rattachent la violence au mécanisme du pouvoir mondial. Plus précisément, elle n'apporte pas d'indication sur les conditions qui permettent la transformation du régime libéral en un régime qui sape les pratiques qui le définissent. Une autre vision sociologique du présent, celle d'Ulrich Beck, également suggestive, va plus loin dans ses descriptions du présent, mais ne contribue pas à une compréhension de la relation entre la violence de la modernité tardive et la politique dans les Etats libéraux et au-delà.

"La nature insaisissable du terrorisme force et permet des constructions d'images de l'ennemi qui ne sont plus limitées par la nature physiquement saisissable des ennemis étatiques. La fusion des concepts d'"ennemi" et de "terrorisme" a ouvert de nouvelles options stratégiques. Les ennemis stratégiques sont à la fois civils et militaires, étatiques et non étatiques, des ennemis territoriaux et non territoriaux omniprésents, d'un point de vue interne comme externe... Tout comme les entreprises peuvent produire des biens trans-localement, les Etats peuvent produire des images changeantes de l'ennemi militairement indépendant de lieux ou d'Etats ${ }^{37}$ ».

C'est crucial car cela suggère "l'élasticité du concept d'ennemi" et génère les conditions dans lesquelles la violence et la force militaire jouent un rôle dominant, là où il n'y a qu'une définition de l'ennemi, où la guerre préventive est légitimée en termes de légitime défense, où un état d'urgence est institutionnalisé tant au niveau interne qu'externe, et où les considération juridiques sont mises de côté au nom de la nécessité ${ }^{38}$. L'analyse de Beck pose problème, en ce qu'elle confine ses commentaires aux Etats-Unis, et c'est la relation entre les réseaux terroristes internationaux et les Etats-Unis qui est au cœur de ses préoccupations.

Par la lecture de telles interprétations de l'ère mondiale actuelle, et de ses manifestations en termes structurels et expérimentaux, on peut s'interroger sur le mode de transformation du régime libéral démocratique, de sorte que les pratiques et les discours de guerre dominent, de sorte que, sans considération pour les luttes historiques pour la contestation et la dissidence, nous entrons dans une phase où ces libertés sont menacées au nom d'insécurités décrétées par l'exécutif. Peut-être les articulations de l'autorité, les mécanismes du pouvoir, tournent-ils entre le répressif et le productif, afin que les deux viennent à s'impliquer et se constituer mutuellement. Là où Foucault cherche à s'éloigner de la souveraineté comme modèle de pouvoir pour se tourner vers celui de la gouvernementalité, plus productif, plus biopolitique, l'analytique de la guerre, elle, éclaire la relation inextricable des deux. Le moment de la guerre est celui des décisions discrétionnaires, celui au cours duquel on désigne les 
ennemis de manière unilatérale. C'est aussi celui où, à l'inverse des souverains des époque précédentes, ceux qui prennent des décisions liées à la vie et la mort d'autres sont des fonctionnaires de l'Etat, d'organisations supranationales comme l'Union européenne ${ }^{39}$, d'entreprises et de groupes privés et semi-privés, ou d'Etat et de groupes de pression, comme c'est le cas de l'actuelle administration Bush. Judith Butler met en lumière la relation de souveraineté et de gouvernementalité, et le fait que «les apparences de souveraineté » prennent forme " dans le champ de gouvernementalité ». Essentiellement, en relation à nos conceptions du régime démocratique libéral, la relation de la décision discrétionnaire et de l'Etat de droit vient à constituer le pilier de la manière dont nous voyons les transformations en cours dans nos sociétés. Pour citer Butler de nouveau:

«Ce n'est pas, pour parler littéralement, qu'un pouvoir souverain suspende l'Etat de droit, mais que cet Etat de droit, en étant suspendu, produit de la souveraineté dans son action et son effet. Cette relation inverse au droit produit la "nonresponsabilité" de cette opération de pouvoir souverain, ainsi que son illégitimitét ${ }^{40} »$.

31 La spatialité globale de la sphère sociale présente des défis particuliers pour toute lecture foucaldienne des mécanismes du pouvoir et de la place qu'y occupe la guerre. Alors que l'analytique de Foucault nous aide à révéler les rouages du pouvoir sur les corps et les populations - des rouages qui, dans cette période moderne, passent de la violence directe des pouvoirs souverains vers des gouvernementalités rationalisantes associées au biopouvoir - il est important de considérer les implications de cette analytique lorsqu'on la considère de manière globale. L'émergence d'une spatialité distinctement globale de la sphère sociale appelle à une lecture et à une appréciation de l'impact différentiel de telles pratiques dans des lieux distincts de la sphère globale. Ces localisations ne sont pas que géopolitiques, ou territoriales, mais elles incluent des lieux associés à l'inégalité dans l'accès aux sources et aux capacités de l'arène mondiale ${ }^{41}$.

32 La compréhension des pratiques d'inclusion et d'exclusion ne peut donc plus se limiter à l'Etat et à ses machineries de contrôle, mais doit s'étendre et inclure les espaces transnationaux d'interaction, comprenant un réseau complexe d'agents, des bureaucraties locales aux institutions internationales. Ces espaces ne peuvent toutefois pas être conçus en termes horizontaux. La manifestation globale de la sphère sociale suggère le global en tant qu'arène de contrôle, ce qui soulève des questions au sujet des agents de contrôle et des possibilités et contraintes qui émergent des inégalités de pouvoir au niveau global. L'émergence du global comme arène de contrôle attire ainsi l'attention vers les technologies capables d'une telle portée, vers les agents qui les possèdent et vers leur volonté et leur capacité à coordonner des services rendus par des acteurs étatiques comme non étatiques. Comme le montre Waltz, bien que trop rapidement, si la plus grande machine de guerre souhaite agir unilatéralement, elle le peut: "Les Etats-Unis ont organisé et contrôlé la campagne en Afghanistan seuls, refusant l'offre de mise à disposition des troupes britanniques du Premier ministre Blair ». Waltz voit le "déséquilibre brut de pouvoir dans le monde» comme une continuité majeure de la politique internationale ${ }^{42}$. Le degré même de ce déséquilibre doit être reconnu lorsqu'on considère la portée globale du pouvoir et, en un sens, les capacités à construire le global comme une arène de contrôle.

33 La spatialité globale de la sphère sociale soulève également des questions substantives quant aux mécanismes associés au contrôle et à la domination. Tandis que la logique 
administrative de l'Etat moderne peut inclure la population nationale dans son champ d'action, dans la situation moderne globalisée, la limite de la population n'est plus coterminus des limites de l'Etat. Notre compréhension de la notion de "frontière " se déplace progressivement du territoire vers le corps. Les implications sont liées à l'expérience phénoménologique du global et de ses inclusions et exclusions, tout comme elles sont liées à la raison administrative de l'Etat moderne et aux relations transnationales institutionnalisées. En d'autres termes, la logique administrative est tournée vers les processus et les dynamiques globaux/transnationaux. Les implications pour le gouvernement et la transparence ("accountability») sont énormes, tant le fonctionnaire qui fait partie des rouages des technologies de contrôle apparaît au-delà de tout soupçon, et donc de toute responsabilité. Alors même lorsqu'un pouvoir discrétionnaire - celui de déclarer la guerre ou, par exemple, d'emprisonner des individus sans respect de leurs droits - fait ou active le moment souverain, le fonctionnaire procède en silence, dans les interstices des pratiques institutionnelles, plaçant certaines catégories de population dans une rationalité calculatrice, elle-même sujette à un profil déterminé par la bureaucratie au-delà du regard des publics et des législatures.

Du point de vue global, les mécanismes associés au contrôle doivent donc être compris à la fois en termes de pratiques institutionnelles de contrôle et en termes de violence associée à la guerre. Lorsque nous considérons l'émergence du global comme arène de contrôle, il ne nous faut pas uniquement nous attarder sur les rouages institutionnels des pratiques gouvernementalisantes mais également sur la capacité qu'ont certains agents à déclarer la guerre, comme manifestation d'un contrôle global. La violence directe qui vise certaines populations en vient à constituer un mécanisme de contrôle pour d'autres populations et gouvernements qui ne sont pas directement visés. C'est en ce sens que la guerre devient une technologie de contrôle.

Les discours qui ont émergé au lendemain des événements du 11 septembre 2001 n'étaient pas simplement des discours sécuritaires, mais aussi des discours de guerre. Comprendre le présent nécessite plus qu'une description des procédures bureaucratiques entreprises à différents niveaux d'interaction domestique et transnationale, des pratiques destinées aux contrôles d'immigration, des mesures de surveillance, etc. Il est certes essentiel de mettre en évidence de telles pratiques, de fournir une image des mécanismes de la gouvernementalité. Mais il est également crucial d'un point de vue critique de localiser de telles pratiques dans un cadre plus large de significations qui nous permet de voir les mécanismes du pouvoir discrétionnaire et les pratiques qui y sont associées, de l'acte de guerre le plus flagrant à la procédure la plus invisible édictée dans un bureau qui se situe hors de notre vue. Un tel cadre nous permet de voir que les pratiques qui constituent la matrice de la guerre sont intrinsèquement liées à la production de différentes formes de sujet et qu'elles sont donc différemment expérimentées.

L'expérience de la matrice de la guerre en vient à dépendre de la localisation du sujet des politiques, localisation qui n'est pas nécessairement définie en termes d'espace géopolitique mais en termes sociétaux et gouvernementalisants, entre le sujet de biopolitiques du présent et son Autre constitutif. Le sujet peut être défini en termes d'espace géopolitique, de classe, de genre, de culture, tous se rejoignant en tant que sites évoqués dans des relations de pouvoir et viennent à constituer les subjectivités du présent ${ }^{43}$. Toutefois, les positions-sujets qui sont produites ou générées dans la matrice 
de la guerre sont en soi toujours changeantes. Les amis d'aujourd'hui peuvent devenir les ennemis de demain dans la position toujours élastique qui prend forme non seulement aux limites de l'Etat mais aussi dans les rues, les villes, les écoles, les immeubles, dans d'autres pays et dans les camps de détention au milieu de ce qui est autrement connu comme des espaces libéraux démocratiques.

L'élasticité de l'ennemi comme position-sujet est toutefois, dans l'arène mondiale actuelle et dans l'Europe d'aujourd'hui, liée aux politiques de la différence, et, plus particulièrement de la différence culturelle. La nature précise de la relation varie selon les contextes et elle est matière à des investigations empiriques. Il est clair que, tandis que les mesures antiterroristes comme le UK Terrorism Act 2000 proscrivent des organisations particulières et possèdent donc un degré de spécificité, le fait que de telles mesures englobent l'élément de "prévention " suggère un cadre de référence plus large, cadre qui, dans les opérations de police, dans les discours institutionnels et dans les arènes publiques, en vient à désigner l'Autre, culturellement marqué comme la source de danger. De plus, lorsque les migrants sont désignés comme des ennemis et sont traités comme tels, il s'agit en général de ceux dont la race et la culture les identifient comme l'Autre. Le racisme en tant que pratique d'Etat atteint les attributions des fonctionnaires, tout comme les décisions discrétionnaires au-delà du droit portent ouvertement et manifestement la marque d'un discours raciste qui vise l'Autre dans son être corporel, que ce soit par le biais d'une forme de colonisation du $\mathrm{xxI}^{\mathrm{e}}$ siècle, comme en Irak ou dans les camps de détention qui contiennent les réfugiés de l'Europe. Comme Michel Foucault l'écrit en relation au passage graduel du XVII' à son présent (et, par voie de conséquence, au nôtre) le « discours de lutte raciale » n'est pas une bataille entre races, "mais à partir d'une race donnée comme étant la vraie et la seule, celle qui détient le pouvoir et celle qui est titulaire de la norme, contre ceux qui dévient par rapport à cette norme $e^{44} 》$.

Les paradoxes ne sont que trop apparents pour l'Etat libéral. Dans les pratiques qui englobent ladite " guerre au terrorisme ", nous voyons les fondements d'une atteinte à la démocratie libérale dans sa conception comme lieu de droits historiquement conquis. Tandis que la violence terroriste cherche précisément une blessure systématique et, par cela, la violation de sociétés aussi différentes entre elles que Londres ou Bagdad, les mesures prises en réponse sont en elles-mêmes des violations de ce que constitue l'espace démocratique et le gouvernement des individus et des communautés.

\section{NOTES}

1.. Cet article a été traduit par Miriam Perier. Il est le résultat de recherches financées par le projet-cadre de la Commission européenne (FP5) intitulé « European Liberty and Security : Security Issues, Social Cohesion and Institutional Development of the European Union : ELISE ». Les passages de ce texte faisant appel à la notion que nous 
appelons la «matrice de la guerre » font référence au texte de Jabri V., «War, Security and the Liberal State ", Security Dialogue, vol.37, $\mathrm{n}^{\circ} 1,2006$.

2.. Voir Waltz K., Man, the State and War : A Theoretical Analysis, New York, Columbia University Press, 1954, ainsi que, du même auteur : Theory of International Politics, Mass, London, Addison-Wesley, Reading, 1979.

3.. Waltz, K. «The Continuity of International Politics », Booth K., Dunne T. (dir.) Worlds in Collision, chapitre 31, London, Palgrave, 2002.

4.. Pour Waltz, la clef est le pouvoir, et, en particulier, le pouvoir militaire. Toutefois, l'élément de " continuité » plus que de transformation est, selon lui, que « les terroristes contribuent à la continuité de la politique internationale. Ils poursuivent des tendances à l'œuvre... le terrorisme est une menace à la stabilité des Etats et à la tranquillité d'esprit de leurs dirigeants... Mais dans la mesure où le terrorisme est une arme brandie par les pauvres, les terroristes ne menacent pas sérieusement la sécurité des Etats». Voir Waltz K., « The Continuity of International Politics », op. cit., p. 353.

5.. Kaldor M., New and Old Wars : Organized Violence in a Global Era, Cambridge, Polity Press, 1999.

6.. Habermas J., The Past as Future, interviewé par Haller M., traduit et édité par Pensky M., Cambridge, Polity Press, 1994, pp. 5-32.

7.. Kant I., « Perpetual Peace : A Philosophical Sketch », Political Writings, Hans Reiss (dir.), traduit par Nisbet H.B., Cambridge, Cambridge University Press, 1970.

8.. Habermas J., Between Facts and Norms, Cambridge, Polity Press, 1997.

9.. Voir Jabri V., «War, Security and the Liberal State », Security Dialogue, op. cit.

10.. Veyne P., « Foucault révolutionne l'histoire », Comment on écrit l'histoire, Paris, Seuil, 1978, rééd. coll. « Points / Histoire », 1996, p. 397.

11.. Voir Jabri V., Discourses on Violence, Manchester, Manchester University Press, 1996.

12.. Sur la question de l'équilibre entre sécurité et liberté dans les discours parlementaires européens, voir Tsoukala A., « Democracy Against Security : The Debates About Counter-Terrorism in the European Parliament ", Alternatives, vol.29, août-octobre 2004, pp. 417-439. Pour une critique philosophique de la notion "d'équilibre », voir Waldron J., " Security and Liberty: The Image of Balance », The Journal of Political Philosophy, vol.11, n²/2003, pp. 191-210.

13.. Voir respectivement Waever O., «Securitization and Desecuritization », Lipschutz R. (dir.), On Security, New York, Columbia University Press, 1995, pp. 46-86 ; Bigo D., "Security and Immigration : Toward a Critique of the Governmentality of Unease ", Alternatives/Cultures \& Conflits, vol. 27, pp. 63-92.

14.. Le rôle de l'Etat en relation à ses citoyens est particulièrement mis en évidence dans le cas de citoyens d'Etats européens incarcérés dans des centres de détention, de Guantanamo à ceux d'Afghanistan et d'Irak.

15.. Deux exemples nous viennent à l'esprit : des hommes politiques italiens de droite qui font référence à des Italiens "bâtards ", et un certain nombre d'objections austroallemandes à l'entrée de la Turquie dans l'Union européenne.

16.. Par exemple, le réseau d'Al Qaeda.

17.. Agamben G., Homo Sacer : Sovereign Power and Bare Life, (trad. Heller-Roazen D.), Stanford, Stanford University Press, CA, 1995. Voir aussi son ouvrage plus récent, State of Exception, Chicago, University of Chicago Press, 2004.

18.. Le Prevention of Terrorism Act 2005 du Royaume-Uni a été accepté le 11 mars 2005, permettant ainsi au Home Secretary d'éditer des « control orders » contre ceux qui ont été « certifiés » terroristes par le Home Secretary. Cette législation remplace l'Anti-Terrorism, 
Crime, and Security Act 2001, qui avait permis au Home Secretary d'incarcérer des citoyens étrangers censés représenter une menace.

19.. Voir Jabri V., op. cit., 2005.

20.. Le soi-disant " principe de précaution » suggère l'idée de prévention pour éviter des risques futurs. C'est devenu un aspect intégral de la prise de décision liée à la gestion et au contrôle du risque, tel que le commente la Commission européenne. Voir le document de la Commission : http://europa.eu.int/comm/dgs/health_consumer/ library/pub/pub07_eu.pdf.

Ce qui est controversé dans le contexte actuel est que ce principe fait partie intégrante de la doctrine de guerre préventive telle que défendue par l'administration Bush et soutenue par le Premier ministre Tony Blair en relation à l'invasion de l'Irak.

21.. Hazel Blears, ministre de l'Intérieur au Home Office. Voir « Muslim Police Stops More Likely », publié sur BBC News, http://news.bbc.co.uk/go/pr/fr/-/1/hi/ uk_ukpolitics/4309961.stm. Publié le 2 mars 2005. Consulté le 28 septembre 2005. 22. Voir Deleuze G., Foucault, (trad. par Hand S.), Minnesota, London, University of Minnesota Press, 1986, pp. 70-93.

23.. Au sujet de l'impact des politiques d'exception sur les démocraties libérales, voir Huysman J., « Minding Exceptions : The Politics of Insecurity and Liberal Democracy », Contemporary Political Theory, vol.3, 2004, pp. 321-341.

24.. Foucault M., Surveiller et punir : naissance de la prison, Paris, Gallimard, 1975.

25.. Foucault M., Il faut défendre la société : cours au Collège de France 1975-1976, Paris, Seuil, 1997.

26. Foucault M., Histoire de la sexualité. La Volonté de savoir, Paris, Gallimard, coll. " Tel », 1994.

27.. Pour une analyse de la structuration de la guerre dans la société, voir Jabri V., Discourses on Violence, Manchester, New York, Manchester University Press, 1996.

28. Foucault M., Histoire de la sexualité. La Volonté de savoir, op. cit, pp. 187-188.

29.. Voir Foucault M., Naissance de la biopolitique : Cours au Collège de France (1978-1979), Paris, Seuil/Gallimard, 2004.

30.. Pour une analyse de la distinction établie par Foucault entre violence et pouvoir et du fait qu'une telle distinction a des implications sur nos préoccupations actuelles en termes de guerre, voir Jabri V., "Critical Thought and Political Agency in Time of War », International Relations, vol.19, n¹, mars 2005, pp. 70-79.

31.. Hardt et Negri, Multitude : War and Democracy in the Age of Empire, Penguin, London, 2004.

32.. Ibid., p. 30.

33.. Voir par exemple Giddens, The Consequences of Modernity, Cambridge, Polity, 1990 ; Castells M., The Information Age : Economy, Society and Culture, Oxford, Basil Blackwell, 1996 ; Beck U., Risk Society: Towards a New Modernity, London, Sage, 1992.

34.. Comme toute analyse néo-libérale de la mondialisation pourrait le suggérer. 35.. Bauman Z., « Reconnaissance Wars of the Planetary Frontierland », Theory, Culture \& Society, vol.19, n4, 2002, p. 81.

36.. Ibid., p. 84.

37.. Beck U., « War is Peace : On Post-National War », Security Dialogue, vol.36, n¹, mars 2005, p. 24. Notre traduction.

38. Ibid., p. 24. 
39.. Voir Bigo D., " Frontier Controls in the European Union : Who is in Control ?", Bigo D., Guild E. (dir.), Controlling Frontiers : Free Movement into and within Europe, Ashgate, Aldershot, 2005.

40.. Voir Butler J., Precarious Life : The Powers of Mourning and Violence, London, Verso, 2004, p. 66.

41.. Concernant les inégalités qui émergent des relations sociales globalisées, voir Bauman Z., Globalisation: The Human Consequences, Cambridge, Polity Press, 1998.

42. Waltz K., « The Continuity of International Politics », op. cit., pp. 349-350.

43.. Voir, par exemple, Jabri V., « Feminist Ethics and Hegemonic Global Politics », Alternatives, vol.29, $\mathrm{n}^{\circ} 3,2004$, pp. 265-284.

44.. Foucault M., Il faut défendre la société, Paris, Seuil, 1997, p. 53.

\section{RÉSUMÉS}

En matière de politique internationale, la guerre est une technologie de contrôle. Tandis que ses manifestations violentes comme l'invasion et l'occupation de l'Irak sont directement ressenties par les populations visées, les pratiques qui y sont associées ainsi que ladite "guerre au terrorisme" ont des effets d'une portée mondiale. Cet article offre une lecture de la guerre globale comme forme de contrôle propre à cette modernité tardive. Il montre que les pratiques qui constituent la guerre globale sont à comprendre en termes de matrice, ceci comprenant les Etats et leurs bureaucraties ainsi que des agents non étatiques, visant Etats, communautés particulières et individus. La matrice de la guerre opère au nom de l'humanité, mais c'est finalement cette même humanité qui en vient à devenir le sujet de ces opérations de contrôle global. Comme le montre l'article, les conséquences sont monumentales pour le gouvernement, l'examen démocratique et les espaces possibles de dissidence.

War in late modern politics is a technology of control. While its violent manifestations, such as the invasion and occupation of Iraq, are directly felt by the population targeted, the practices associated with this and the wider so-called war against terrorism, have a far wider span of operations that encompasses spaces across the globe. This article provides an understanding of global war as a distinctly late modern form of control. It shows that the practices constitutive of global war are best understood in terms of a matrix, incorporating states and their bureaucracies as well as non-state agents, and targeting at once states, particular communities, and individuals. The matrix of war operates in the name of humanity ; however, it is ultimately this humanity as a whole that comes to be the subject of its operations of global control. The implications, as the article argues, are monumental for democratic government and the spaces available for scrutiny and dissent.

\section{INDEX}

Mots-clés : biopouvoir, guerre, Relations Internationales, Théorie politique 


\section{AUTEURS}

VIVIENNE JABRI

Vivienne Jabri est maître de conférences au centre d'Etudes des Relations internationales du Department of War Studies, King's College à Londres. Elle participe aux programmes de recherche européens ELISE et CHALLENGE. Ses publications (dont Discourses on violence et un certain nombre d'articles) développent une lecture critique de la guerre et de ses relations au politique. Elle rédige un ouvrage sur la modernité tardive et les politiques de la violence. 